\title{
Changes in tissue oxyhaemoglobin concentration measured using multichannel near infrared spectroscopy during internal carotid angiography
}

\author{
T J Germon, P E Evans, N J Barnett, T T Lewis, P Wall, R J Nelson
}

\begin{abstract}
Objective-To develop an in vivo model for testing spatially resolved spectroscopy and quantified near infrared spectroscopy (NIRS) cerebral blood flow measurements.

Method-Multiple detector NIRS has been used to study changes in tissue oxyhaemoglobin $\left(\mathrm{O}_{2} \mathrm{Hb}\right)$ concentration during selective internal carotid angiography. A significant reduction in $\mathrm{O}_{2} \mathrm{Hb}$ occurred in tissue interrogated by detectors situated between 0.7 and $4.1 \mathrm{~cm}$ from the NIRS light source.

Results-The time course of $\mathrm{O}_{2} \mathrm{Hb}$ concentration change was consistent with displacement of oxygenated blood by the radiocontrast medium from vascular beds of differing flow and NIR light attenuation. Increasing changes in $\mathrm{O}_{2} \mathrm{Hb}$ concentration per unit photon path lengthpredicted to occur at greater emitterdetector separations if those changes had occurred predominantly in cerebral tissue-were found in the first four seconds after injection of radiocontrast medium. However, later changes (6-10 s) were larger and were not proportional to emitter-detector separation.

Conclusion-The findings indicate that simple assumptions regarding the distribution of the internal carotid artery blood supply to cerebral and extracerebral tissues, the photon path length through those tissues, and their relative contributions to attenuation of NIR light may not be justified.
\end{abstract}

(F Neurol Neurosurg Psychiatry 1997;63:660-664)

Neuroradiology,

Frenchay Hospital, Bristol BS16 1LE, UK

T T Lewis

Johnson and Johnson Medical Ltd, Imperial House, Imperial Way, Celtic Lakes, Newport, Gwent NP1 9UH, UK

P E Evans

N J Barnett

$\mathrm{P}$ Wall

Correspondence to:

Dr TJ Germon, Department of Neurosurgery, Frenchay Hospital, Bristol BS16 1LE, UK.

Received 23 September 1996 and in revised form 25 April 1997

Accepted 18 June 1997 dura from those taking place in the cerebrumthat is, to achieve spatial resolution-depend on the use of dual channel NIRS sensors. Experimental work supporting this approach and subsequent clinical studies have assumed that occluding ${ }^{910}$ or injecting indocyanine green into the internal carotid artery ${ }^{11}{ }^{12}$ will result in purely cerebral changes in NIR light attenuation. To validate this assumption and to develop an in vivo model for testing spatially resolved spectroscopy and quantified NIRS cerebral blood flow measurements we have examined changes in tissue oxyhaemoglobin $\left(\mathrm{O}_{2} \mathrm{Hb}\right)$ concentration during selective internal carotid angiography using multichannel NIRS.

\section{Method}

In its simplest form NIRS applies the BeerLambert Law to light attenuation in tissue to calculate the concentration of absorbing compounds (chromophores). The presence of multiple chromophores necessitates the use of multiple interrogating wavelengths to determine the concentration change of each individual chromophore, the general methodology of which has been described elsewhere. ${ }^{13}$ For this study a prototype multichannel NIR spectrometer (Johnson and Johnson Medical Ltd, UK) employing four laser diodes operating at $777,819,871$, and $909 \mathrm{~nm}$, sequentially pulsed at $912 \mathrm{~Hz}$ was used. These wavelengths are maintained with a Peltier device feedback loop controlling the laser diode temperature and each light pulse is $100 \mathrm{~ns}$ in duration with a peak power output of $10 \mathrm{~W}$. Two metres of fibre optic cable conducts the NIR light from the spectrometer to a silicon rubber sensor where the light emitting window is mounted together with eight high sensitivity photodiodes. The photodiode detectors are mounted in a linear array at $0.7,1.3,2.0,2.7,3.4,4.1,4.8$, and 5.5 $\mathrm{cm}$ (channels 1-8 respectively) from the emitter. The signals detected in each channel are amplified, integrated, and converted from an analogue to digital format before being processed by an algorithm based on absorption spectra for the chromophores $\mathrm{O}_{2} \mathrm{Hb}$, deoxyhaemoglobin ( $\mathrm{HHb})$, total haemoglobin $(\mathrm{tHb})$, and oxidised cytochrome C oxidase (Cyt) obtained in non-scattering media ${ }^{14}$ and incorporating corrections for wavelength dependent differences in scattering. ${ }^{16}$ The multicomponent algorithm produces numerical values for $\mathrm{O}_{2} \mathrm{Hb}, \mathrm{HHb}, \mathrm{tHb}$, and Cyt. As these values are calculated using a pathlength of unity, changes are proportional to changes in 


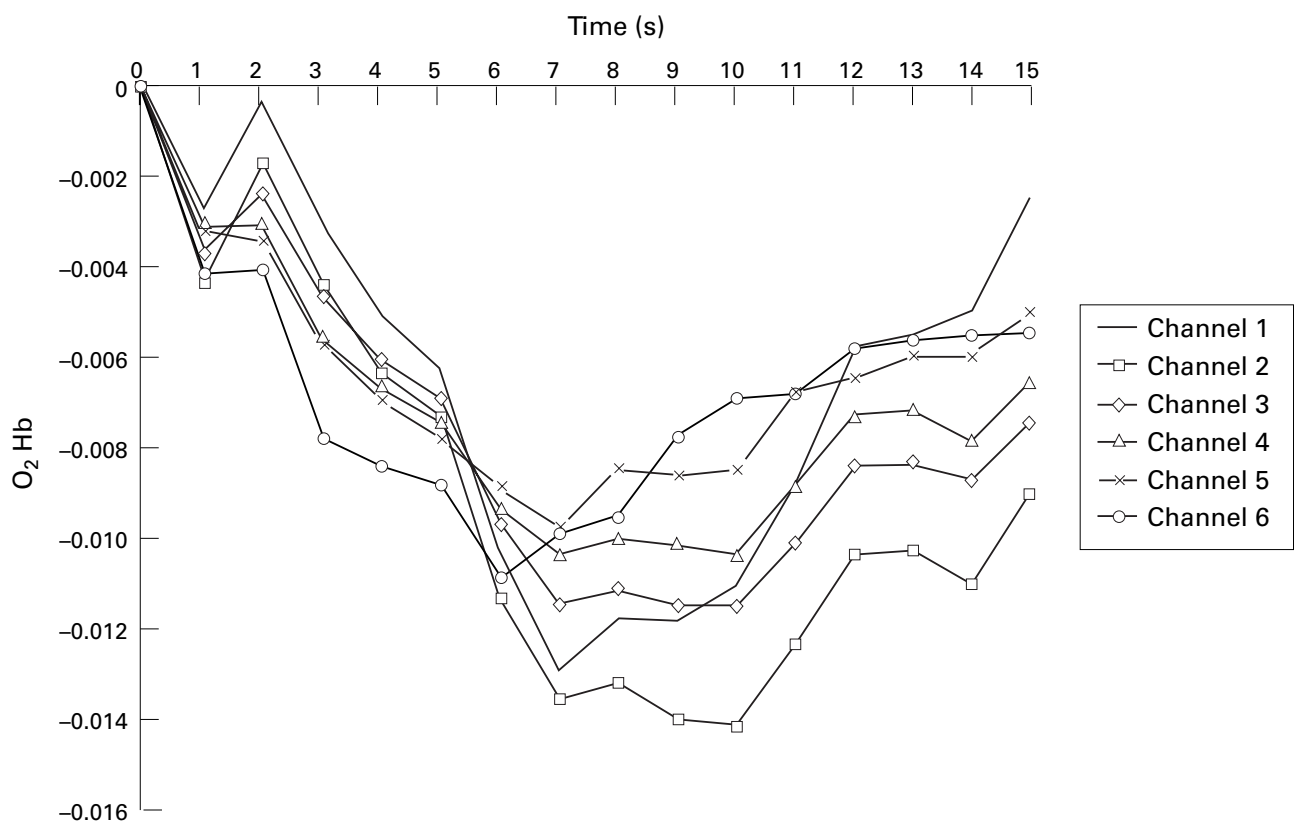

Figure 1 The mean change in $\mathrm{O}_{2} \mathrm{Hb}$ concentration per unit path length $\left(\mathrm{O}_{2} \mathrm{Hb}\right.$, $\mu m o l l^{-1} \times$ differential pathlength factor (DPF)) for each second after the injection of contrast for channels 1-6 (channels 7 and 8 are omitted for clarity).

concentration multiplied by the photon path length through the tissue.

The multichannel sensor was attached with a self adhesive pad to the skin of the left or right frontal region of eight consenting patients (six men and two women, mean age 42 years) undergoing digital subtraction cerebral angiography using a Phillips Integris C2000 angiography unit for the investigation of subarachnoid haemorrhage or epilepsy. The sensor was placed with the light emitter just medial to the superior temporal line, as close to the coronal suture as the hairline would allow with the detectors lying anteromedially. The patients were premedicated with morphine $(10-15 \mathrm{mg})$ intramuscularly. In two patients bilateral studies were performed. The internal carotid artery was selectively catheterised using a 5 French Mani catheter introduced by a standard transfemoral percutaneous route and $8 \mathrm{ml}$ contrast media (Ultravist 300, Schering AG, Germany) were hand injected over about 1.5 seconds. We previously established that the contrast medium is transparent to light in the NIR part of the electromagnetic spectrum (unpublished data). The time of the start of injection was recorded with an event marker.

The absolute change in the $\mathrm{O}_{2} \mathrm{Hb}$ signal detected in each channel was divided by the distance between the emitter and detector for that channel to yield a figure proportional to the $\mathrm{O}_{2} \mathrm{Hb}$ concentration change per $\mathrm{cm}$ of emitter-detector separation. The change in $\mathrm{O}_{2} \mathrm{Hb}$ concentration every second for 15 seconds after the injection of contrast, was plotted for each channel. The significance of the mean change in $\mathrm{O}_{2} \mathrm{Hb}$ in the 10 seconds immediately after the injection was compared with 10 seconds of baseline recording before the angiographic injection was determined using a paired Student's $t$ test. A P value $<0.01$ was considered significant, allowing for multiple comparisons. Differences in the time course of changes detected in each channel were then compared by analysis of variance (ANOVA). If significant differences were detected, Newman-Kuel's test was used to determine the significance of differences at individual time points.

\section{Results}

The results of NIRS monitoring of 10 lateral angiographic studies were analysed. One was omitted from further comparisons because event markers failed to be recorded. Figure 1 shows the time course of the mean reduction in $\mathrm{O}_{2} \mathrm{Hb}$ concentration for channels 1-6 for each emitter-detector separation after the angiographic injection. Channels 7 and 8 have been omitted for clarity because of low signal to noise ratios and wide between patient variations. The median time of maximum reduction in $\mathrm{O}_{2} \mathrm{Hb}$ concentration occurred at six seconds. Compared with the respective values 10 seconds before injection the mean reductions in $\mathrm{O}_{2} \mathrm{Hb}$ concentrations in the 10 seconds after injection were highly significant in channels $1-6(P<0.001$, fig 2$)$. The mean reductions in channels 7 and 8 were of similar magnitude,

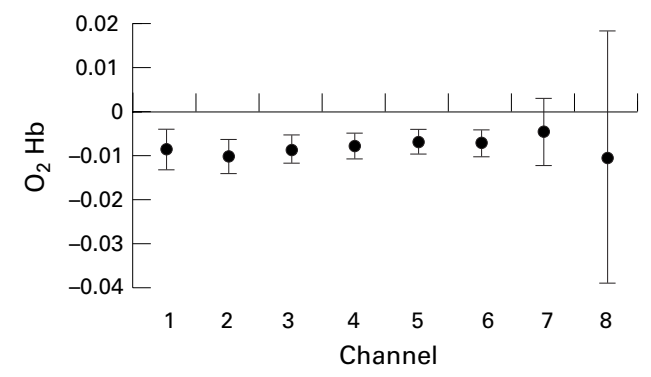

Figure 2 The mean change in $\mathrm{O}_{2} \mathrm{Hb}$ concentration per unit photon path length $\left(\mathrm{O}_{2} \mathrm{Hb}\right.$, $\left.\mu \mathrm{mol} l^{-1} \times \mathrm{DPF}(\mathrm{SD})\right)$ between the 10 seconds before injection and the 10 seconds immediately after contrast injection for channels 1-8. A P value $<0.01$ was accepted as significant to allow for multiple comparisons. 
but were not significant due to the variance of the data (channel $7 \mathrm{P}=0.371$; channel 8 $\mathrm{P}=0.020$ ). No significant difference was found between the responses of channels 1-6 (fig 2). However, multivariate ANOVA disclosed significant differences in the time course of changes detected in channels 1-6. Further tests of significance using Newman-Kuel's test at individual time points indicated that the change in channel 6 was significantly greater than channels 1,2 , and 3 at three seconds and significantly greater than channel 1 at four seconds. Conversely, the change at nine and 10 seconds was significantly greater in channels 1 and 2 than channels 5 and 6 .

\section{Discussion}

The inherent time resolution of NIRS is ideally suited to the detection of transient changes in cerebral blood flow and oxygenation. As high quality cerebral angiography depends on the momentary displacement of oxygenated blood from the cerebral arterial bed we predicted that this study would show a significant reduction in $\mathrm{O}_{2} \mathrm{Hb}$ concentration in those channels interrogating cerebral tissue.

Theories of light propagation in tissue suggest that as the distance between emitter and detector is increased the mean tissue sample depth is also increased. ${ }^{17-19}$ Thus it has been assumed that increasing the distance between emitter and detector will result in a greater proportion of attenuation taking place in cerebral tissue than in extracerebral tissue. Therefore, if internal carotid angiography transiently alters $\mathrm{O}_{2} \mathrm{Hb}$ concentration in the cerebral vascular bed alone we would anticipate increasing attenuation with increasing emitter-detector separation.

When comparing data measured at different emitter-detector separations it is important to normalise for differences in the predicted optical path length as attenuation will naturally be greater for larger separations. In this study all changes in concentration were divided by the distance between the emitter and detector in $\mathrm{cm}$. This correction assumes that there is a linear relation between the optical path length in tissue and the emitter-detector separation. Although this is almost certainly an oversimplification of the true behaviour of NIR light in the adult, until that behaviour is fully understood, a linear correction remains the best available. The concentration change per unit photon path length could be further divided by the differential pathlength factor (DPF), which describes the relation between emitter-detector separation and actual photon path length. ${ }^{20}$ This would yield a figure for the average absolute concentration change $\left(\mu \mathrm{mol} \mathrm{l}^{-1}\right)$ in the tissue volume interrogated. However, whereas determinations of the DPF by time of flight or other techniques provide an estimate of how far the photons have travelled through the head, ${ }^{20}$ they do not provide any indication of where the photons have been. If only a small proportion of the total distance travelled by the photons has been through cerebral tissue, using a DPF determined by these methods to estimate changes in cerebral chromophore concentra- tion will produce erroneous results. Therefore, we have elected not to use such constants until their validity has been established.

The detection of significant changes in $\mathrm{O}_{2} \mathrm{Hb}$ concentration in response to the internal carotid artery injection confirms the sensitivity of NIRS to changes in tissue oxygenation and $\mathrm{Hb}$ content. However, changes detected in the short range channels were unexpected findings which must ultimately reflect similar degrees of NIR light attenuation taking place per $\mathrm{cm}$ emitter-detector separation in each channel.

There are several possible explanations for this finding. Part of the optical path of the short range channels may pass through the cerebrum and the change in $\mathrm{O}_{2} \mathrm{Hb}$ concentration in cerebral tissue in response to the angiographic injection may be so great that it overwhelms the effects of extracranial NIR light attenuation at all emitter-detector separations. Although this explanation would be at odds with generally accepted models of NIR light behaviour we have found significant changes in $\mathrm{O}_{2} \mathrm{Hb}$ concentration in all eight channels of the same prototype cerebral oximeter in response to a hypocapnic test of cerebrovasular reactivity, ${ }^{21}$ implying that either the scalp responds to changes in $\mathrm{pCO}_{2}$ in a similar fashion to cerebral tissue (for which there is some evidence ${ }^{22}$ ) or, indeed that small emitter-detector separations are sensitive to changes in cerebral oxygenation.

The time course and pattern of change in $\mathrm{O}_{2} \mathrm{Hb}$ concentration detected in channels 1-6 support the possibility of NIR light attenuation taking place in vascular beds with differing transit times. There was a significantly greater reduction in channel 6 detected at three and four seconds after injection of radiocontrast. This reduction is consistent with displacement of oxygenated blood from the cerebral capillary bed supplied by the internal carotid artery and supports the hypothesis that sensitivity to spectroscopic changes in cerebral tissue is increased at longer emitter-detector separations. However, maximal reductions occurred six to seven seconds after injection into the internal carotid artery and changes detected at 9 and 10 seconds after injection were significantly greater in the proximal channels. This is consistent with a reduction in $\mathrm{O}_{2} \mathrm{Hb}$ concentration in tissues of differing vascular transit times throughout their arterial, capillary, and venous phases. If this explanation is correct, the fourfold greater change in $\mathrm{O}_{2} \mathrm{Hb}$ concentration occurring in the slow phase compared with the fast phase, with a reversal in the relation between the size of change and emitterdetector separation, indicates that extracerebral changes in NIR light attenuation play a major part. In our study $8 \mathrm{ml}$ contrast medium were injected at pressures equal to or above systemic arterial pressure into the internal carotid artery. The injections were selective with the catheter tip placed several $\mathrm{cm}$ above the carotid bifurcation and there was no radiological evidence of contrast entering the external carotid artery. Although reflux into the external carotid artery circulation remains a possibility, it is unlikely to be the sole explanation for our findings. 
The attenuation of NIR light by adult extracerebral tissues may be more relevant than previously thought; perhaps as a result of photon channelling at multiple tissue boundaries considered by Okada et al. ${ }^{23}$ Although their model does not account for the multiple tissue layers of the skin, scalp, and skull it does show that a single tissue layer could have a profound effect on the mean light path of NIR photons. If this were the case changes in $\mathrm{O}_{2} \mathrm{Hb}$ concentration in blood supplied to the scalp and diploe of the frontal region from the internal carotid artery via the ophthalmic and supraorbital arteries ${ }^{24}$ may predominate over cerebral changes at least for emitter-detector separations up to $4.1 \mathrm{~cm}$. The persistence of attenution changes beyond the end of the angiographic phase is difficult to explain and raises the possibility of secondary vascular or autoregulatory events.

McCormick et al injected $1 \mathrm{ml}$ indocyanine green (ISG) solution into the internal carotid artery while the external carotid artery was occluded to prevent reflux. ${ }^{11}$ As in our study, selective injection into the internal carotid artery resulted in a significant increase in attenuation at $1 \mathrm{~cm}$ and $2.7 \mathrm{~cm}$ with maximum increases in attenuation occurring six to eight seconds after injection and remaining significantly raised for more than one minute after injection. However, by contrast with our findings, the increase at $1 \mathrm{~cm}$ was significantly smaller than that detected at $2.7 \mathrm{~cm}$. This discrepancy may be explained by the different analysis of spectroscopic data in the two studies. We corrected for the greater photon path length between the emitter detector by dividing changes by the emitter-detector separation whereas McCormick et al used the raw data in their analysis.

In a similar study Hongo et $a l^{12}$ examined uncorrected attenuation changes detected between 1 and $4.5 \mathrm{~cm}$ from an NIR emitter after ICG injection into the internal carotid artery and external carotid artery during selective cerebral angiography. Again, significant increases in attenuation were detected at $1 \mathrm{~cm}$, which increased with emitter-detector separation, with maximum changes seen at six to eight seconds after injection. Conversely, after injection into the external carotid artery there was no difference in absolute change in attenuation detected at increasing emitter-detector separation. If these data were expressed as change per unit photon path length, the results of internal carotid artery injection would be comparable with ours whereas a decrease in attenuation with increasing emitter-detector separation comparable with that predicted by theoretical models for a change in extracerebral oxygenation and found in response to scalp ischaemia, ${ }^{25}$ would be described.

Until the relative contributions of the internal carotid artery and external carotid artery to the blood supply of extracranial tissues have been established, studies which assume that changes found in response to manipulations of the internal carotid artery reflect solely cerebral disturbances must be interpreted carefully; particularly in patients with advanced occlusive arterial disease who commonly have complex collateral anastomoses between the internal carotid artery and external carotid artery. On the other hand, if the internal carotid artery does not supply extracerebral tissue, the evidence suggests that even $1 \mathrm{~cm}$ emitter-detector separations are sensitive to changes in cerebral NIR attenuation. If this is the case attempts to increase sensitivity to cerebral attenuation changes by increasing emitter-detector separation may be futile as any theoretical advantage is outweighed by increasingly poor signal to noise ratios.

This study, together with work showing the sensitivity of NIRS to changes in extracranial blood flow, ${ }^{6-8}$ raises questions about the quantification of NIRS measurements made with a single light source and receiver with no attempt at spatial resolution. Studies which correlate changes in NIRS with middle cerebral artery blood flow velocity or other cerebral ${ }^{26}$ or systemic ${ }^{27}$ indices may be heavily influenced by the anatomy of the blood supply to the frontal region.

This study confirms the sensitivity of NIRS to changes in tissue $\mathrm{Hb}$ concentration as well as the potential for both spatial and temporal resolution of transient change, at the same time as highlighting the complexities of the interaction between perfusion of cerebral and extracerebral tissue and the attenuation of NIR light.

TJG was supported by the Stroke Association. This work was presented in part to the Joint meeeting of the British and North American Neurosurgical Research Societies, Newcastle, May 1996 and to The International Society on Oxygen Transport to Tissue, Dundee, Scotland, August 1996.

1 Mason PF, Dyson EH, Beard JD. The assessment of cerebral oxygenation during carotid endarterectomy utilising near infrared spectroscopy. Eur F Vasc Surg 1994;8:590-4.

2 McCormick PW, Stewart M, Goetting MG, Dujovny M, Lewis G, Ausman JI. Non-invasive cerebral optical spectroscopy for monitoring cerebral oxygen delivery and haemodynamics. Crit Care Med 1991;19:89-97.

3 Williams IM, Vohra R, Farrell A, Picton AJ, Mortimer AJ, McCollum CN. Cerebral oxygen saturation, transcranial Doppler ultrasonography and stump pressure in carotid Doppler ultrasonography and stump
surgery. Br $\mathcal{F}$ Surg 1994;81:960-4.

4 Slavin KV, Dujovny M, Ausman JI, Hernadez G, Luer M, Slavin KV, Dujovny M, Ausman JI, Hernadez G, Luer M,
Stoddart H. Clinical experience with transcranial cerebral Stoddart H. Clinical experience with trans
oximetry. Surg Neurol 1994;42:531-40.

5 Mead GE, Williams IM, McCollum CN, Mortimer AJ. Near infrared spectroscopy-a method for continuous measurement of cerebral oxygenation. British fournal of Intensive Care 1995; June:194-9.

6 Germon TJ, Kane NM, Manara AR, Nelson RJ. Near infrared spectroscopy in adults: effects of extracranial ischaemia and intracranial hypoxia on estimation of cerebral oxygenation. Br $\mathcal{F}$ Anaesth 1994;73:503-6.

7 Harris DNF, Cowans FM, Wertheim DA. NIRS in the temporal region-strong influence of the external carotid
artery. In: $\mathrm{P}$ Vaupel, et al, eds. Oxygen transport to tissue $X V$. Adv Env Med Biol 1994;345:825-8.

8 Germon TJ, Young AER, Manara AR, Nelson RJ. Extracerebral absorption of near infrared light influences the detecebral absorption of near infrared light influences the detec-
tion of increased cerebral oxygenation monitored by near infrared spectroscopy. $\mathcal{F}$ Neurol Neurosurg Psychiatry 1995; 58:477-9.

9 W8:477-9. McCollum CN. Cerebral hypoxia detected by near infrared spectroscopy. Anaesthesia 1994;49:762-6.

10 Kirkpatrick PJ, Smielewski P, Whitfield PC, Czosnyka M, Menon D, Pickard JD. An observational study of near infrared spectroscopy during carotid endarterectomy. $\mathcal{F}$ Neurosurg 1995;82:756-63.

11 McCormick PW, Stewart M, Lewis G, Dujovny M, Ausman JI. Intracerebral penetration of infrared light. $\mathcal{F}$ Neurosurg 1992;76: 315-8.

12 Hongo K, Kobayashi S, Okudera H, Masonobu H, Nakagawa F. Non-invasive cerebral optical spectroscopy:
depth-resolved measurements of cerebral haemodynamics depth-resolved measurements of cerebral haemodyn
using indocyanine green. Neurol Res 1995;17:89-93.

13 Cope M, Delpy DT. A system for long term measurement of cerebral blood and tissue oxygenation in newborn infants by near infrared illumination. Med Biol Eng Comput 1988;26:289-94. 
14 Wray JS, Cope M, Delpy DT, Wyatt JS, Reynolds ER Characterisation of the near infrared absorption spectra of
cytochrome aa 3 and haemoglobin for the non-invasive monitoring of cerebral oxygenation. Biochem Biophys Acta monitoring of cere

15 Brunori M, Antonini E, Wilson MT. Cytochrome oxidase: an overview of recent work. In: H Sigel, ed. Metal ions in biological systems. New York: Marcel Dekker, 1981:188228 .

16 Essenpreis M, Cope M, Elwell CE, Arridge SR, van der Zee P, Delpy DT. Wavelength dependence of the differentia pathlength factor and the log slope in time-resolved tissue spectroscopy. In: U Dirnagl, et al, eds. Optical imaging of brain function and metabolism. Adv Exp Med Biol 1993;333. 9-20.

17 Nossal R, Nossal RF, Havlin S, Weiss J. Model of photon migration in turbid biological tissue. $\mathcal{F}$ Opt Soc Am 1987;4: migration

18 Cui W, Kumar C, Chance B. Experimental study of migration depth for the photons measured at sample surface. tion depth for the photons mea
Proc SPIE 1991;1431:180-91.

19 Cui L,Ostrander L. Assessment of layered optical properties in biological tissue from dual wavelength optical reflectance measurement. Proceedings of the $12^{\text {th }}$ Annual International Conference IEEE Engineering In Medicine and Biology Society. IEEE Trans Biomed Eng 1990;12:1115-6.

20 Delpy DT, Cope M, van der Zee P, Arridge S, Wray S, Wyatt J, Estimation of optical pathlength through tissue from direct time of flight measurement. Phys Med Biol 1988;33:1433-42.
21 Germon TJ, Evans PD, Barnett NJ, Wall P, Nelson RJ. The effect of increasing optode separation on the sensitivity of near infrared spectroscopy to intra- and extracranial oxygenation changes. F Cereb Blood Flow Metab 1995; 15(suppl 1):617.

22 Barker SJ, Hyatt J, Clarke C, Tremper K. Hyperventillation reduces transcutaneous oxygen tension and skin blood flow. Anaesthesiology 1991;75:619-24.

23 Okada E, Firbank M, Schweiger M, Arridge SR, Cope M, Delpy DT. A theoretical and experimental investigation of the effect of sulci on light propagation in brain tissue. Proc SPIE 1995;2626: 2-8.

24 Williams PL, Warwick R, Dyson M, Bannister LH. eds. Gray's anatomy, 37th ed. London: Churchill Livingstone, 1989.

25 Germon TJ, Evans PD, Barnett NJ, Wall P, Manara AR, Nelson RJ. Sensitivity of near infrared spectroscopy to intra- and extracerebral oxygenation changes is determined by optode separation. Clinical Intensive Care 1995;6:198.

26 Elwell CE, Owen-Reece H, Cope M, Wyatt JS, Edwards AD, Delpy DT, Reynolds EO. Measurement of adult cerebral haemodynamics using near infrared spectroscopy. Acta Neurochir 1993;59(suppl):74-80.

27 Roberts I, Fallon P, Kirkham FJ, Lloyd-Thomas A, Cooper C, Maynard R, et al. Estimation of cerebral blood flow with near infrared spectroscopy and indocyanine green. Lancet 1993;342:1425. 